\title{
NANO-MATERIALS FOR THE CONSERVATION AND PRESERVATION OF MOVABLE AND IMMOVABLE ARTWORKS
}

\author{
P. Baglioni a, *, R. Giorgi a, D. Chelazzi a \\ a Department of Chemistry and CSGI, University of Florence, via della Lastruccia 3, Sesto Fiorentino, 50019 Florence, Italy - \\ (baglioni, giorgi, chelazzi)@esgi.unifi.it
}

KEY WORDS: Conservation, nanomaterials, compatibility, durability, cleaning, deacidification, consolidation, nanoparticles, gels, low environmental impact

\begin{abstract}
:
In the context of the FP7 NANOFORART project (Nano-materials for the conservation and preservation of movable and immovable artworks) new nano-materials and responsive systems have been developed and experimented for the preservation of works of art. Conservation of cultural heritage is still mainly based on traditional methods and conventional materials that often lack the necessary compatibility with the original artworks and a durable performance in responding to the changes of natural environment and manmade activities. The main challenge of NANOFORART is the combination of functional materials arising from the recent developments in nano-science with innovative techniques in the restoration of works of art. The research activity has been focused on the development of manageable methodologies, based on nanosized structures and with a low environmental impact.

The main tasks include the production of dispersions of nanoparticles, micellar solutions, microemulsions and gels, in order to offer new reliable pathways to restore works of art by combining the main features of soft and hard-matter systems for cultural heritage conservation. Great importance was also given to technology transfer to SMEs that will play an important role in the standardization of applicative protocols, in the up-scale and commercialization of technology and in the evaluation of the eco-toxicity of nanomaterials. Important museums, such as the National Museum of Anthropology and History of Mexico City and the National Museum of Denmark, are validating the technology developed in the project, and providing training activities and dissemination of the developed techniques.
\end{abstract}

\section{INTRODUCTION}

\subsection{Objectives}

The FP7 NANOFORART project is focused on the development and application of novel nanomaterials and nanostructured responsive systems for the conservation and preservation of moveable and immoveable artworks. The project is coordinated by CSGI (Consorzio Interuniversitario per lo Sviluppo dei Sistemi a Grande Interfase - Center for Colloid and Surface Science) and includes a wide partnership of experienced Institutions, museums and enterprises.

As a matter of fact, while the progress in Materials Science has already produced sophisticated nanostructured materials, the conservation of cultural heritage is still mainly based on traditional methods and conventional materials that often lack the necessary compatibility with the materials constituting the original artworks and a durable performance in response to changes of environmental conditions caused by natural events or anthropic activities.

The main challenge of NANOFORART is thus the integration of sophisticated functional materials arising from the recent developments in Nano-science/technology with innovative and ad hoc designed techniques in the restoration and preventive conservation of works of art, with unprecedented efficiency.

On one hand, the research activity will be focused on the development of new materials and, on the other hand, on the design and optimization of suitable and durable conservation methods in order to provide restorers and end-users with innovative but manageable methodologies, based on nanostructured systems and with a low environmental impact. The project will thus be focused on the production of dispersions of nanoparticles, micellar solutions, microemulsions and gels, to be used according to new reliable pathways to restore and preserve works of art by combining the main features and properties of soft and hard-matter systems, allowing the design of specifically tailored chemical tools for cultural heritage conservation and preservation.

While assessment of the developed technology on real and widely representative conservation case studies and issues will be performed, the overall efforts will also be directed to technology transfer to SMEs and end users, that will play an important role in the assessment and refinement of the developed nanotechnology, in the standardization of applicative protocols, in the dissemination of the developed techniques and in the evaluation of the human health effects and environmental impacts of nanomaterials.

The general objectives of the NANOFORART project are summarized in the following points:

1. Design and formulation of "smart" nanostructured systems with special functionalities, which will include:

- deacidification of movable artworks (paper, parchment, textile, leather);

- cleaning of movable artworks (paper, parchment, canvas paintings);

- consolidation of immovable artworks (wall-paintings, plaster

\footnotetext{
* Corresponding author.
} 
and stones);

- cleaning of immovable artworks (wall-paintings, plaster and stones);

with particular attention to the control of chemical functions in order to maximize the compatibility of materials with the treated artworks, and to the minimization of the use of high environmental impact products. The advanced nanomaterials and techniques (cleaning, consolidation, deacidification, $\mathrm{pH}$ adjustments) that will be developed within the project are compatible with the physico-chemical characteristics of artworks, and will secure the conservation of the original objects and/or improve the physical state of the damaged objects.

2. Physico-chemical characterization of the systems in order to understand and control the nature of the mechanism of interaction between the nanostructures and the target substances and supports.

3. Assessment of the applicability of materials in order to evaluate the contribution to preventive conservation and thus, implicitly, to the sustainability and authenticity of the cultural assets. In this phase a first scale-up of the technologies from the laboratory to the market level will be tackled. All the partners will interact in order to clarify and merge the priority from all the points of view.

4. Study of the long-term behaviour of the products and of the treated works of art by means of accelerated and natural ageing, in order to avoid damages due to unforeseen phenomena. The partners have their main competence in accelerated ageing of conservation materials, monitoring of environmental pollution, in particular emissions of volatile organic acids, and of art objects on display in museums and in storage. The proposal will thus assess the long-term behaviour of the materials as regards the durability of the historic assets and/or performance of the chosen techniques.

5. Development of industrial processes for the transfer of technology to market by the standardization of the applicative protocols and production of the nanomaterials on medium and large scale. Small and Medium Enterprise (SME) partners have their main competence in this area.

6. Evaluation of possible human health effects and environmental impact of nanomaterials for restoration. The technology generated by NANOFORART will be investigated with special emphasis on the potential hazardousness of nanoparticles used for design and formulation of nanostructured system and the environmental impact associated with the usage of developed nano-based products. Nanotechnology will significantly reduce the use of solvents and will introduce new environmentally friendly materials. The potential for adverse environmental and health impacts relating to the new materials and/or techniques will thus be assessed.

7. Dissemination of the developed technologies and training activities. In this phase a network of end users (e.g. museums, restoration public and private bodies, SMEs) will be built. End users will be active in both education and training activities as well as in the final refinement of the developed technology.

\section{CONCEPTS}

\subsection{Progress beyond the state-of-the-art}

Nanotechnology is revolutionizing material science in a pervasive way. The continuous development of novel nanostructure-based materials and the study of physicochemical phenomena at the nanoscale are introducing new approaches to conservation science, leading to new methodologies that can slow down the degradation processes of works of art or even recover them from the damages introduced by detrimental restoration campaigns.

In the past few years CSGI (coordinator of NANOFORART project) has pioneered the development of new technologies for artworks preservation and conservation and has provided restorers with novel scientific and technological solutions to restoration and conservation problems (Baglioni 2006).

Within the NANOFORART project, new nanomaterials will be developed and assessed, both through restoration expertise by restorers, end-users and SMEs partners, and advanced analytical methods by partners as scientific centres and universities, in order to address different restoration issues involving conservation and preservation of movable and immovable artworks.

State-of-the-art nano-thermal analysis will be used for the first time in conservation science, in order to characterize the developed nanomaterials and their effects on the treated artworks. In the particular case of collagen-based artefacts, the proposal will benefit from the expertise of recent revisions of collagen structure at the nanoscale level. The structure has been described in terms of nanoscale ropes, providing new insight into collagen fibril structure (Bozec, 2007).

In the following sections important examples of the state-of-theart knowledge in preservation and conservation of movable and immovable artworks, and the progress proposed by the NANOFORART project, will be shown, focusing on cleaning, consolidation and deacidification procedures.

\subsection{Cleaning of immovable artworks}

The state-of-the-art in cleaning procedures of natural or synthetic degraded and detrimental materials, such as adhesives, coatings, consolidants and protectives, is mainly related to the use of organic solvents and solvent gels (Wolbers 1989).

The traditional solvent cleaning is based on the identification of the materials to be removed, and on the selection of the solvent blend that maximizes the cleaning action. Teas charts are used to predict the power of solvent mixtures (Phenix 1998, Hiemenz 1997).

One of the issues in solvent cleaning of degraded natural or synthetic coatings is the loss of solubility of these materials due to molecular weight changes following their natural aging.

Another important problematic drawback related to the state-ofthe-art use of solvents for cleaning immovable artworks such as wall-paintings, stone, plaster and stucco, is the re-dissolution of the natural or synthetic material into the porous matrix of the artefact.

Moreover, the usage of pure organic solvents increases the toxicity and environmental impact of the cleaning techniques.

The usage of solvent gels, first advocated in the 1990s, allows the localization of the solvent and, in some cases, the reduction of solvent's penetration into underlying paint layers. Unfortunately, it is not always easy to remove solvent gels and their residues from a paint surface and this problem will also be addressed in this proposal.

Recently, nanocompartmentalized systems tailored either for the specific removal of Paraloid B72 or of Mowilith DM5 (vinyl 
acetate/n-butyl acrylate $65: 35 \mathrm{w} / \mathrm{w}$ copolymer) resins from wall paintings, have been formulated by Carretti et al. (Carretti 2003). These systems are oil-in-water microemulsions (that from now on will be referred to as nanocontainers) or micellar solutions that ensure low aggressiveness with respect to the original components of the painted layer due to the presence of water as a dispersing medium that remains in direct contact with the hydrophilic surface of the wall painting. The large surface area of nanocontainers and micelles accounts for the strong interaction with the polymer coating, resulting in high detergency capability. Nanocontainers (whose dimensions are about tens of nanometers) provide a high solving power of the coating's layers, while micelles (whose dimensions are less than $10 \mathrm{~nm}$ ) provide a fast swelling and detachment of the coating's layers at the interface between the coating's surface and the surface of the micelles. The detached coating's layers are then carried away in the micellar solution and hence removed. Both the solubility power and the swelling/detachment action are key-features for an improved cleaning and removal of the degraded coatings.

Compared to the traditional method based on pure solvents, nanocontainers and micellar solutions are very promising and offer better and faster performance, and decreased eco-toxicity and health risks, due to the fact that the percentage of pure organic solvents used in these formulations is usually less than $5 \%$ (water is the major solvent component of these systems, with a mass content $\geq 95 \%$ ).

Another advantage granted by water-based systems is the reduced penetration into the immovable artwork's porous matrix with respect to the one that occurs with organic solvents. Hence, the polymer re-dissolution into the artefact is avoided.

Progress beyond the state-of-the-art in the NANOFORART project focused on the design and development of nanostructured fluids, such as nanocontainers and micellar solutions, that are specifically tailored for the simultaneous extraction of blends of different synthetic polymers or of blends of synthetic and natural organic materials used in past restorations.

In fact, Paraloid B72 and Mowilith DM5 have been very often used in mixtures or sequentially applied to wall paintings, so an ideal system should remove simultaneously both resins, in onestep cleaning process. Also blends of natural and synthetic materials are common in the state-of-the-art conservation practice, and this can have a detrimental result on artworks.

Oil-in-water $(\mathrm{o} / \mathrm{w})$ microemulsions, based on an anionic surfactant (sodium dodecyl sulphate) and 1-pentanol, have been obtained by using propylene carbonate and ethyl-acetate as a co-solvents.

The design of efficient formulations for the tailored removal of polymers requires a deep comprehension of the solubilization mechanisms, which can be more complex than traditional detergency, due to the presence of partially water-soluble organic solvents (cosolvents). One of the key features is the size of the nanocontainers and micelles: a smaller size results, for a given surfactant content, in a higher interfacial area that is correlated to the kinetics of the cleaning process.

The design process has been thus monitored by using scattering techniques (quasi-elastic light scattering, QELS, and smallangle neutron scattering, SANS) to optimize the size and the shape of nanocontainers and micelles, in order to achieve the best cleaning performance. Analytical investigations also granted a better understanding of the removal mechanisms and of the interactions between the nanostructured fluids and the artefacts. In particular, Atomic Force Microscopy (AFM) has been used to follow the first steps of solubilisation process at the nanoscale.

Parameters such as penetration in the artworks layers, the removal rate, the amount of removed materials and of left residues (surfactants) have been also investigated basing on the analysis of treated models and real samples.

Different cleaning case-studies of immovable artworks have been selected and faced, ranging from Northern to Eastern Europe and Mesoamerican areas. The selection was based on the representativeness criterium: the conservation issues considered in this project are, in fact, representative of the majority of real conservation case-studies.

\subsection{Consolidation of immovable artworks}

As discussed in the previous section, the most common practice in preservation of immovable artworks is nowadays based on the use of synthetic organic materials that very often are not compatible with the physico-chemical properties of the original artworks.

Given all the issues arising from the degradation of these coatings and from the lack of physicochemical compatibility with the substrates they are used on, the cleaning step is often a necessary preliminary phase for immovable artworks treated with such consolidants as acrylic or vinyl polymers.

After the cleaning, a new consolidation intervention, using compatible materials, will provide a more durable and compatible preservation and conservation of the artworks.

In the case of recently uncovered and yet untreated immovable artworks, on the other hand, consolidation is often the first step, previous to cleaning procedures.

The usage of compatible nanomaterials for consolidation of immovable artworks has been pioneered by the work of P. Baglioni et al. (Ambrosi 2001, Giorgi 2010a).

Materials such as calcium and magnesium hydroxide exhibit high compatibility with the surface of many immovable artefacts such as wall-paintings and frescoes, and represent an innovative and promising alternative to the usage of synthetic coatings.

Calcium hydroxide particles can be transformed into a material with excellent features as a consolidation agent, when synthesised as a nanomaterial.

In fresco painting consolidation, for example, nanoparticles of calcium hydroxide efficiently interact with carbon dioxide to reform calcium carbonate and replace the degraded original ligand, leading to the re-cohesion of the paint layer (Ambrosi, 2001).

In the NANOFORART project, progress beyond the state-ofthe-art has consisted in the development of novel tailored nanoparticle-based materials for consolidation of wall paintings, stones, plaster and stucco.

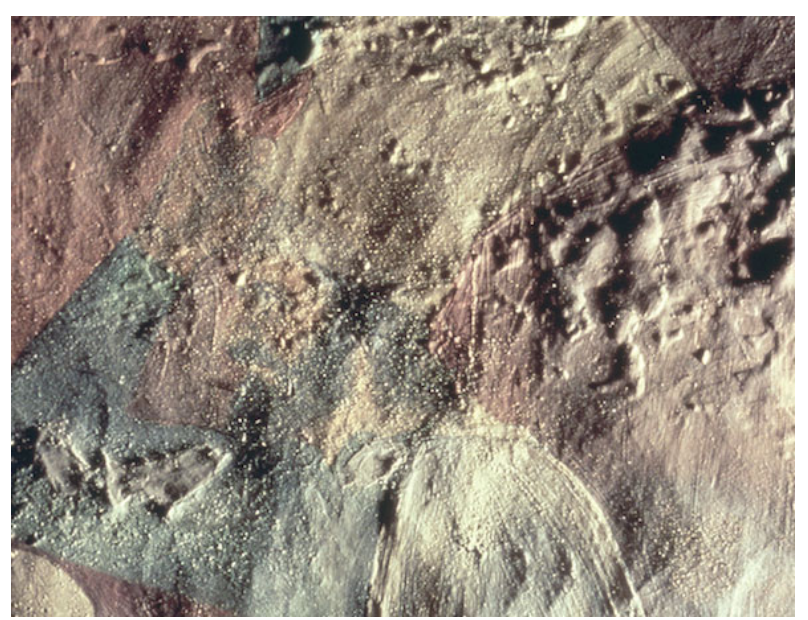




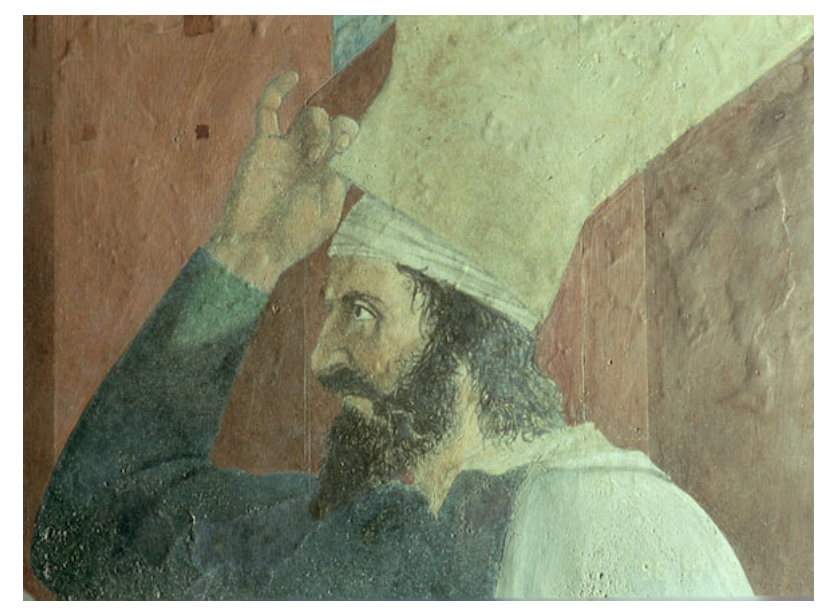

Figure 1. Scene from the "The Story of the True Cross" by Piero della Francesca ( $15^{\text {th }}$ century), in Arezzo (Italy). On top, a pre-restoration image of the wall-painting. On bottom, a postrestoration image. Cleaning of sulphates and consolidation were performed by using the Ferroni-Dini method (Baglioni 2006).

The great diversity in the composition of artworks substrates, in fact, usually require the development of different formulations in order to achieve the maximum compatibility, both in terms of chemical composition and porous structure. Thus, each formulation have required specific procedures for the synthesis. For example, in the presence of large amounts of sulphates (as contaminants), the consolidation effect of calcium hydroxide (that is the original binder of mural paintings) was enhanced by the complementary use of barium hydroxide nanoparticles, which leads to the formation of the totally insoluble, and therefore inert, barium sulphate (Giorgi 2010b) (figure 1).

For this purpose, it was necessary to synthesize particles with the same average size and highly similar particle size distribution, in order to avoid their clustering and separation. A top-down approach (by using high-energy milling) was followed to prepare barium hydroxide nanoparticles with average size of about $250 \mathrm{~nm}$ and size distribution compatible with calcium hydroxide particles, which are commonly synthesized through a homogenous phase reaction (bottom-up approach)

Progress beyond the state-of-the-art will be thus aimed at the design and upscale of several formulations of nanomaterials, that can be used for different consolidation issues, granting an easy use by restorers and a low environmental impact. Prototypes developed as demonstrators (not directly commercialized) in the framework of the project will hopefully provide fundamental information for possible (future) commercial exploitation of these technologies, specifically matching the requirements of conservation end-users.

As for the cleaning section, representative and world-wide transferable case-studies have been fundamental for the development of effective consolidation treatments for immovable artworks. Successful applications of mixed formulation of calcium and barium hydroxide have been recently done in the Maya site of Calakmul (Mexico).

\subsection{Cleaning of movable artworks}

Moveable works of art to be considered in this proposal include two broad categories: cellulosic (painting canvases, paper) and collagen-based materials (parchment and leather). Cleaning of moveable objects such as painting canvases, paper, parchment and leather artworks is particularly challenging since their physical and mechanical properties deteriorate with aging.
In the case of canvas-supported paintings, the state-of-the-art in cleaning procedures of the painted surface is mainly related to the use of solvent gels coupled with a wide range of solvents. The efforts in developing cleaning strategies have thus been pointing towards a complete and selective removal of dirt, degraded coatings, varnishes or adhesives and other detrimental materials, without affecting the underlying artwork's surface and bulk layers.

In order to obtain a better control on the cleaning action of solvents, gels and poultices have been increasingly developed in the last decades. Solvent gels developed by Wolbers, employing partially deprotonated (carboxylate/carboxylic acid forms) poly(acrylic acid)s (PAcAs) as gellants, have been the most frequently used to remove varnishes (Wolbers 1989). The application of solvent gels is relatively easy and their effectiveness is often high, but they might leave residues on artworks that are hard to clean without damaging the substrate's surface (Stulik 2004).

Starting from the Wolbers ideas, the need of new formulations has arisen in order to get a full control in the confinement of either pure solvents (as happens in Wolbers' systems) and the more efficient and responsive nanostructured cleaning agents (micellar solutions, nanocontainers). Chemical and physical gels have been developed in order to sequester (rather than to gel) cleaning agents.

A new formulation of highly elastic viscous poly(vinylalcohol) polymeric dispersion (HVPD) for conservation applications by Carretti et al., enables the modulation of the HVPD adhesion to the art object by controlling the HVPD structure and its degree of cross-linking. After the cleaning action, the HVPD can be easily peeled from the painted surface without the addition of a second liquid component, as is necessary with the Wolbers solvent gels (Carretti 2009, Carretti 2010).

Other useful formulations for chemical gels include the recent development of nanomagnetic sponges by Bonini et al.. By adding magnetic nanoparticles to the gel's polymeric network, it is possible to make the gel responsive to an external magnetic field (Bonini 2007), allowing the complete removal of the cleaning gel from the painted surface without leaving residues and avoiding additional contact between the conservator and the artwork. The mesh size of the polymeric network (usually inhomogeneous domain sizes of a few tens of nanometers, and micrometric pores) can be controlled by tuning the induced cross-linking, granting control over the compartmentalization of the cleaning liquid phase that is embedded in the gel.

Highly viscoelastic HVPDs and magnetic gels are very promising materials for cleaning movable artworks surfaces, but many questions remain about the range of their applications and the long term consequences of using them on artworks.

Progress beyond the state-of-the-art in the NANOFORART project have been focused on developing new chemical gel formulations, starting from the latest results, in order to make them effective in a wide range of different applications for cleaning movable artworks.
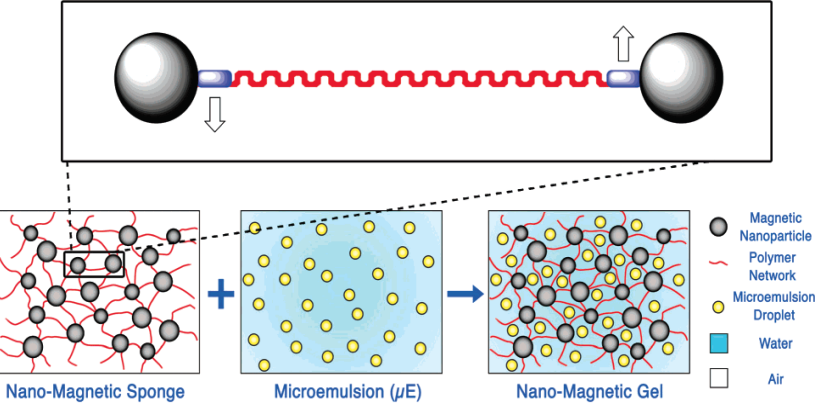

IMMOVABLE ARTWORKS

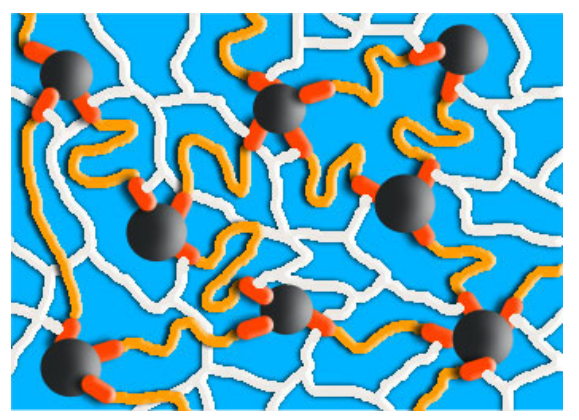

Figure 2. Schematic representation of the process of loading a microemulsion into an acrylamide-based polymeric gel structure. (top) Representation of cross-linked nanoparticles (black spheres) bonded to residues of maleic anhydride (blue rectangles) and a polyethylene glycol chain (red line). Arrows represent the binding group to the acrylamide-based polymer network. (bottom) The gel-particles network. Adapted with permission from (M.Bonini, S.Lenz, R. Giorgi, P.Baglioni, Nanomagnetic sponges for the cleaning of works of art, Langmuir). Copyright (1007) American Chemical Society

Diversification in the formulation of gels will be performed in order to adapt them to the sequestration of different nanostructured cleaning agents (nanocontainers, micellar solutions) and solvents.

The designed cleaning systems have been differentiated in order to achieve the maximum compatibility with the surfaces they were applied on. High-polarity cleaning systems (like hydrogels, oil-in-water nanocontainers or water-based micellar solutions), that work very efficiently for the removal of organic coatings from wall paintings, have been adapted in order to avoid drawbacks when applied on paper, canvas, parchment and leather (e.g. swelling of fibers).

High retention capability provided by chemical gels has prevented from risks of swelling and weakening of water sensitive support,s during cleaning.

Cleaning of canvas has been focused on tow main topics: cleaning of painted surfaces (canvas paintings, easel paintings) and cleaning of canvas surfaces treated with adhesives (lined canvas).

In order to design the cleaning systems for canvas paintings, traditional painting varnishes have been considered, including natural varnishes (e.g. dammar, shellac, sandarac) and synthetic (e.g. regalrez, hydrocarbon resin).

In order to design the cleaning systems for canvas surfaces treated with adhesives, both real and model samples treated with adhesives, natural glues (e.g. animal glue, waxes, resins, "colle de pate") and synthetic adhesives (acrylic and vinyl copolymers) have been considered.

Investigations on the topology of the gel action (i.e., the depth and rate of cleaning action, especially as measured quantitatively and in situ) have been performed, by using electron (FEG-SEM) and atomic force microscopy (AFM).

As previously reported for cleaning of immovable artworks, widely representative case-studies have been faced in the development of cleaning tools for movable artworks: painted silk textiles, painted globes (treated in the past with adhesives), and poor tempera' canvas paintings. Preliminary testing have provided really encouraging results. The preparations here described will be also tested on samples, which will be made available from the previous EU project IDAP (Improved Damage Assessment of Parchment). The advantage of testing methodology on such samples is that knowledge is available on the physico-chemical state of these samples, also at the nanoscale level from atomic force microscopy (AFM) images and then quantification of these images, also from the IDAP project. Similarly leather samples will be provided by the School of Conservation, Copenhagen, which remain from the previous STEP Leather project.

\subsection{Deacidification of movable artworks}

As a matter of fact, acidity is one of the main causes of moveable artworks degradation.

It has recently been highlighted that paper objects are deteriorating rapidly because of, mainly, two interconnected degradation pathways: the acid hydrolysis of glycosidic bonds and oxidation (Wouters 2008).

The state-of-the-art in paper deacidification treatments includes aqueous and non-aqueous methods, the latter being usually less invasive since it limits or avoids the swelling of cellulose fibres. Among the non aqueous methods, the Bookkeeper (Preservation Technologies, L.P.) is an effective method based on dispersion of mainly micro-sized particles of $\mathrm{MgO}$ in fluorinated solvents. This method presents several advantages, but also some drawbacks: when paper porosity is low a light whitening of the document surface may be produced due to the fact that the particles present in the Bookkeeper are not small enough to completely penetrate inside the paper. Moreover, in order to stabilize the magnesium oxide dispersion, high concentrations of fluorinated surfactants are used. These surfactants remain on paper and their long-term effects are not yet known (Zumbühl 2001).

Recent studies (Giorgi 2005) have shown that alcoholic dispersions of calcium and magnesium hydroxide nanoparticles can be used for the neutralization of paper acidity and can generate an alkaline reserve of carbonate (after the reaction of the hydroxide with $\mathrm{CO}_{2}$ from air) that prevents further degradation.

This method has been so far positively welcomed. The main advantages rely in the nanosized particles that grant a good penetration inside the paper fibers and a quick carbonation due to their high surface reactivity. Moreover, no surfactants are used to stabilize the alkaline nanoparticles.

It has also been shown (Kolar 2003) that the catalytic activity of copper and iron ions is minimal when $\mathrm{pH}$ is around neutrality. This means that the decrease of the degradation rate of oxidation through Fenton reactions could be provided by a precise control of paper acidity/alkalinity.

When dealing with highly oxidised paper, however, it is particularly important to adjust $\mathrm{pH}$ around not too high values, in order to avoid alkaline degradation of the aged cellulose fibres.

As a progress beyond the state-of-the-art, the NANOFORART project has aimed at developing new nanomaterials for deacidification of movable artworks, and testing their performance, applicability and durability.

The most important advance is represented by the upscale of the materials and the development of prototypes as demonstrators (not directly commercially exploitable), which can provide the basis for future commercialisation and the implementation of the methodology in semi-industrial mass-treatments.

Synthesis and preparation of nanoparticles are in many cases time-consuming and big limitations in terms of reaction yield are usually shown. Recently developed procedures for calcium and magnesium hydroxides exhibit these drawbacks that make them not well adaptable to large-scale production.

In the framework of NANOFORART project new chemical routes, based on a bottom-up approach, have been investigated with very important results. Particles with a narrow size distribution and average size about $100 \mathrm{~nm}$ have been obtained. Particular interest has also been focused in less aggressive formulations based on calcium and magnesium carbonates, in 
order to gain the proper $\mathrm{pH}$ adjustment and to avoid alkalinity excess.

The concept of $\mathrm{pH}$-adjustment to reach the ideal value for conservation is also particularly important when dealing with leather and parchment treatments.

In fact, it is well known that a high level of acidity is detrimental to such artworks, promoting degradation of the protein molecules of collagen through hydrolytic processes, but also alkalinity, on the other hand, could result in damaging the fibres.

For deacidification of movable artworks, highly representative case-studies have been faced in the context of the NANOFORART project. Special emphasis has been given to modern and contemporary manuscripts; in these cases, the presence of solvent soluble inks strongly limits the possibility to perform deacidification treatment. The selection of proper solvent blends, which are compatible with ink materials, has provided good results for a wide range of documents.

\subsection{References}

Ambrosi, M. (et al.), 2001. Colloidal particles of $\mathrm{Ca}(\mathrm{OH})_{2}$ : Properties and applications to restoration of frescoes. Langmuir 17, pp. 4251-4255.

Baglioni, P. (et al.), 2006. Soft and hard nanomaterials for restoration and conservation of cultural heritage. Soft Matter 2, pp. 293-303.

Bonini, M. (et al.), 2007. Nanomagnetic Sponges for the Cleaning of Works of Art. Langmuir 23, pp. 8681-8685.

Bozec, L., 2007. Collagen fibrils: Nanoscale Ropes". Biophysical Journal 92(1), pp. 70-75.

Carretti, E. (et al.), 2003. Solubilization of acrylic and vinyl polymers in nanocontainer solutions. Application of nanocontainers and micelles to cultural heritage conservation", Langmuir 19, pp. 7867-7872.

Carretti, E. (et al.), 2009. Poly(vinylalcohol)-borax hydro/cosolvent gels. Viscoelastic properties, solubilizing power, and application to art conservation. Langmuir 25, pp. 8656-8662.

Carretti, E., (et al.), 2010. New Frontiers in Materials Science for Art Conservation: Responsive Gels and Beyond. Accounts of Chemical Research 43(6), pp. 751-760.

Giorgi, R. (et al.), 2005. Nanoparticles of $\mathrm{Mg}(\mathrm{OH})_{2}$ : synthesis and application to paper conservation. Langmuir 21, pp. $8495-$ 8501 .

Giorgi, R. (et al.) 2010a. New Methodologies for the Conservation of Cultural Heritage: Micellar Solutions, nanocontainers, and Hydroxide Nanoparticles. Accounts of Chemical Research 43, pp. 695 -704.

Giorgi, R. (et al.) 2010b. Nanoparticles for Cultural Heritage Conservation: Calcium and Barium Hydroxide Nanoparticles for Wall Painting Consolidation Chem. Eur. J. 16, pp. 93749382 .

Hiemenz, P.C., 1997. "Principles of Colloid and Surface Chemistry”, Marcel Dekker: NewYork, 1997.

Kolar, J. (et al.), 2003. Stabilisation of corrosive iron gall inks. Acta Chim. Slov. 50, pp. 763-770.
Phenix, A., 1998. Solubility parameters and the cleaning of paintings: An update and review. Z. Kunsttechnol. Konserv. 12, pp. 387-409.

Stulik, D. (et al.), 2004. Solvent Gels for Cleaning of Works of Art. The Residue Question. Dorge,V. (Ed.), The Getty Conservation Institute: Los Angeles, 2004.

Wolbers, R.C., 1989, Notes for Workshop on New Methods in the Cleaning of Paintings". Getty Conservation Institute: Los Angeles, 1989.

Wouters, J., 2008. Coming soon to a library near you? Science 322, pp. 1196-1198.

Zumbühl, S. (et al.), 2001. Chemical Aspects of the Bookkeeper Deacidification of Cellulosic Materials: The Influence of Surfactants. Studies in Conservation 46, pp. 169-180. 